\title{
THE SWOLLEN LEG
}

D T Reilly, John H N Wolfe

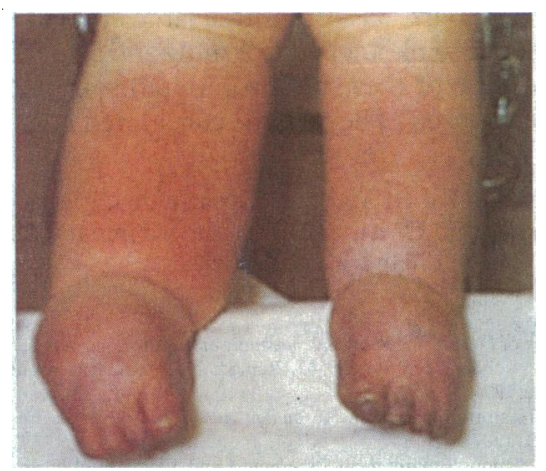

Dependent oedema in a patient with poliomyelitis.

\section{Pathophysiology}

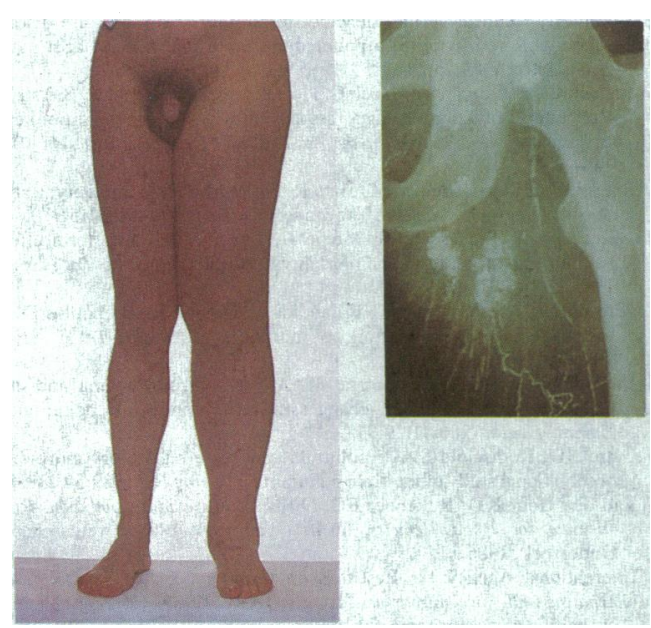

Pelvic lymphatic obstruction shown clinically (left) and radiographically (right) with a profusion of abnormal lymphatics running into inguinal lymph nodes but no lymphatics running into the pelvis.

\section{Clinical features}

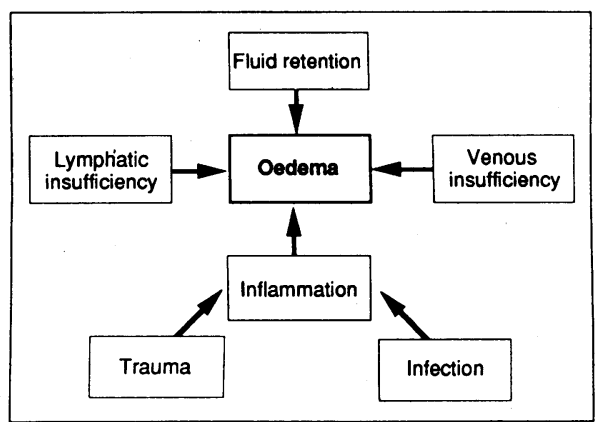

In both hospital and general practice the patient with a swollen leg presents a common dilemma in diagnosis and treatment. The cause may be trivial or life threatening - an insect bite or a deep vein thrombosis - and it is difficult to negotiate the path between overinvestigation and laissez faire.

Fortunately in most cases the decision becomes clear after a careful history has been taken and a clinical examination carried out.
It is helpful to return to simple physiological concepts to understand the development of swelling of the lower limb. In a normal limb the hydrostatic pressure of the column of blood in the veins is balanced by the calf muscle pump, which depends for its efficiency on the integrity of the venous valves as well as on the ability of the muscle to contract. Thus several causes of swelling are immediately apparent: postphlebitic damage to valves, prolonged dependency (as in a leg with rest pain), or failure of the muscle pump (as in a paralysed limb or an arthritic limb with fixity of the ankle joint). The period of rest and elevation of the limb every night normally permits resolution of any slight oedema that may have built up during the day: if this normal behaviour is prevented (as during a prolonged flight) oedema results.

The redistribution of fluid from the arterial end of the capillary to the venous end (Starling's law) explains the mechanisms of the production of oedema. Venous hypertension causes increased pressure in the postcapillary venule and thus back diffusion of fluid into the tissues; lower plasma oncotic pressure also prevents tissue fluid returning to the vein from the interstitial space. Increased capillary permeability (as accompanies injury or allergy) will cause oedema by exudation of fluid rich in protein into the tissue spaces. Failure to remove protein - and therefore fluid-because of lymphatic insufficiency is the other main cause of oedema.

Causes of both acute and chronic swelling of the leg can be grouped into: - General-fluid retention as in cardiac and renal failure, and hypoproteinaemia

- Local-venous or lymphatic insufficiency

- Inflammatory - allergy, trauma, or infection.

Much can be gained from examination of the affected limb, particularly the pattern of the swelling: if the oedema is maximal in the ankles and legs, and the feet are spared, the cause is more likely to be venous than lymphatic. 


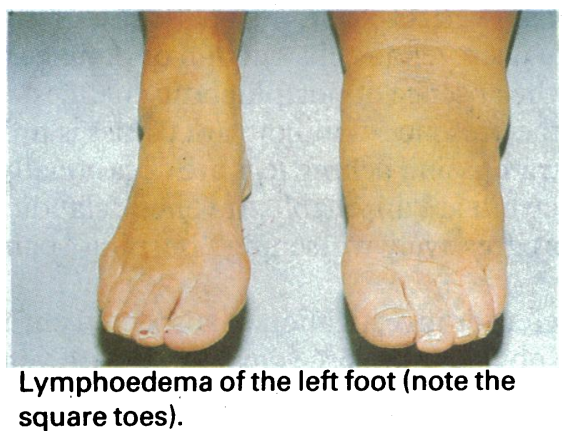

\section{The acutely swollen leg}
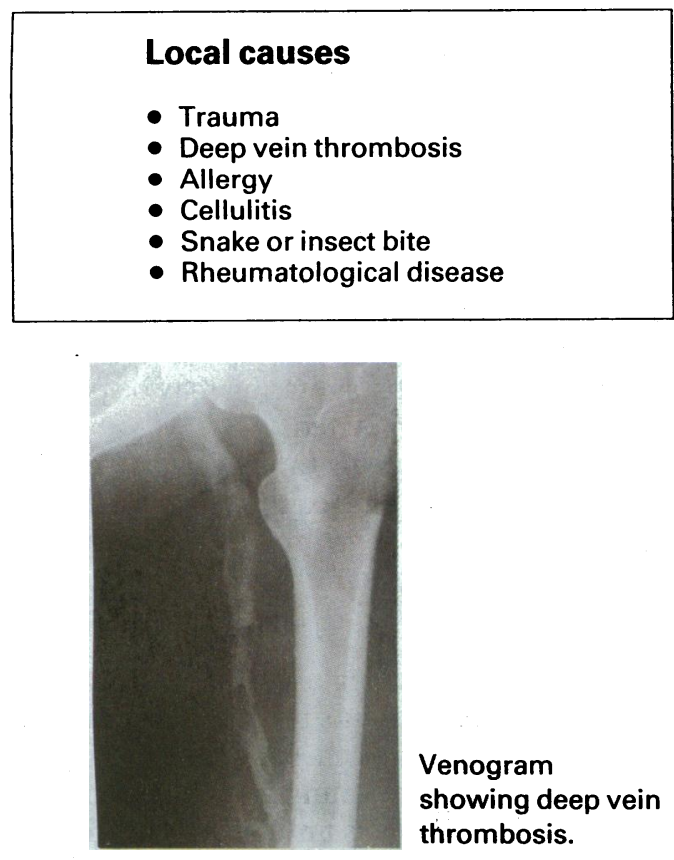

Venogram

showing deep vein thrombosis.

\section{The chronically swollen leg}

\begin{tabular}{|l|}
\hline Local causes \\
- Venous: \\
Postphlebitic limb \\
Venous compression \\
Varicose veins \\
- Lymphoedema: \\
Primary \\
Secondary \\
- Congenital malformation \\
- Dependency
\end{tabular}

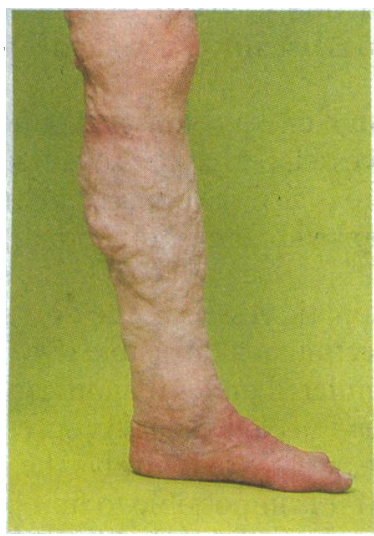

Swelling caused by venous disease.
Deposition of haemosiderin and atrophic or ulcerated skin also favour a venous cause, whereas diffuse long standing oedema that is most prominent distally with hypertrophied lichenified skin is the picture of lymphoedema. It is often stated that cardiac oedema pits and lymphatic oedema does not, but all oedema pits initially and becomes brawny as fibrin is deposited in the intercellular spaces. We will consider here only local causes, assuming that systemic causes have been excluded.

Trauma and allergy, insect bite and cellulitis, can rapidly be differentiated. Examination should show a portal of entry and should also pick up such traumatic causes as undisplaced fracture (bony tenderness) or haematoma (bruising and fluctuance). Rheumatological causes should be suspected if there is a joint effusion or a skin rash (for example, erythema nodosum), and a ruptured Baker's cyst is usually easy to feel. Lymphoedema is painless unless there is associated cellulitis.

The cause that cannot confidently be diagnosed or excluded on clinical grounds is the most worrying - that is, deep vein thrombosis. It is still sometimes asserted that clinical signs are enough, and consequently some patients without deep vein thrombosis receive long term anticoagulant treatment because of a confident clinical diagnosis. Sufficient studies have now been carried out to show that $20-30 \%$ of the patients with signs and symptoms will fail to show a deep vein thrombosis on venography. Conversely, exclusion of deep vein thrombosis by clinical examination alone is wrong half the time. Characteristically an acute deep vein thrombosis (if it causes clinical signs) produces tense swelling of the deep compartment and not just superficial oedema. Management of deep vein thrombosis is discussed in the article on thrombosis and pulmonary embolism.

The mainstay of treatment of patients with chronically swollen legs (once systemic causes have been excluded) is compression with elastic stockings, so it may be argued that there is little point in investigation if the treatment is to be the same. This is a counsel of despair: there are a number of surgical options available for a subgroup of patients with venous or lymphatic insufficiency, and this minority will be denied adequate treatment if the right diagnosis is never made. Prognosis depends on diagnosis-for instance, the patient with lymphoedema will be advised to have immediate treatment with antibiotics for minor infections of the affected limb to prevent loss by fibrosis of further scarce lymph vessels.

\section{History and examination}

The common causes of chronic swelling are venous and lymphatic insufficiency; several other causes can, however, be diagnosed on clinical grounds alone. For example, a common source of confusion is arterial insufficiency; a patient immobilised with rest pain may sleep in a chair or leave his or her foot dangling over the side of the bed, so that the most obvious feature is oedema of the foot and leg. Here the history and pain in the calf on dorsiflexion of the foot are usually enough to make the diagnosis. Similarly, the immobility of a patient with a cerebrovascular accident may lead to oedema in the affected leg that may simulate a deep vein thrombosis.

Venous causes - A history of deep vein thrombosis should always be sought, but will often be non-existent even in the presence of the typical appearance of the leg with chronic venous insufficiency - that is, a swollen, brawny, pigmented calf with eczema or ulcers. There may be obvious varicose veins. Occasionally primary varicose veins can be the cause of 


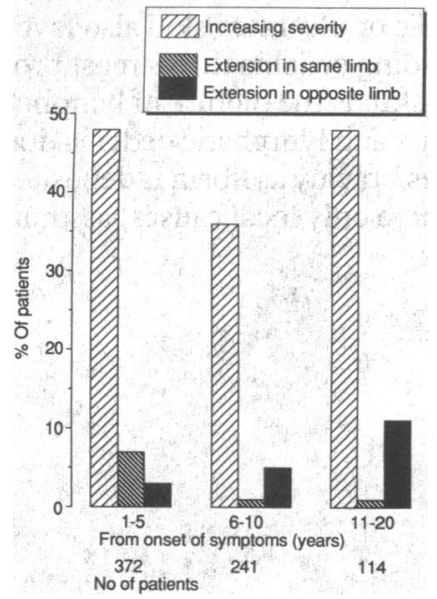

Progress of lymphoedema.

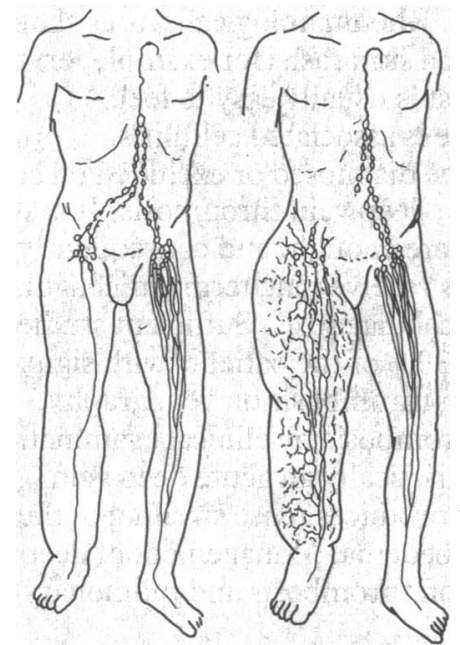

Distal lymphatic obliteration (left) and pelvic obstruction (right). Distal obliteration is much more common among women, and is mild. Pelvic obstruction occurs in twice as many women as men, and is severe.

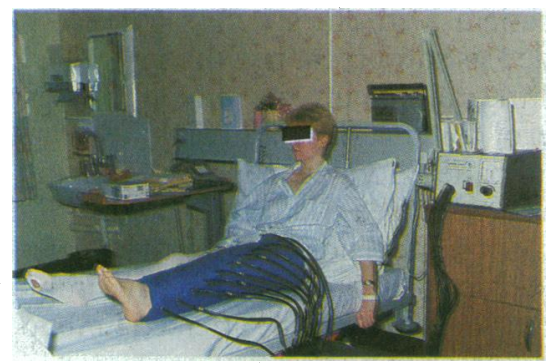

Lymphoedema being treated with a Lymphapress.

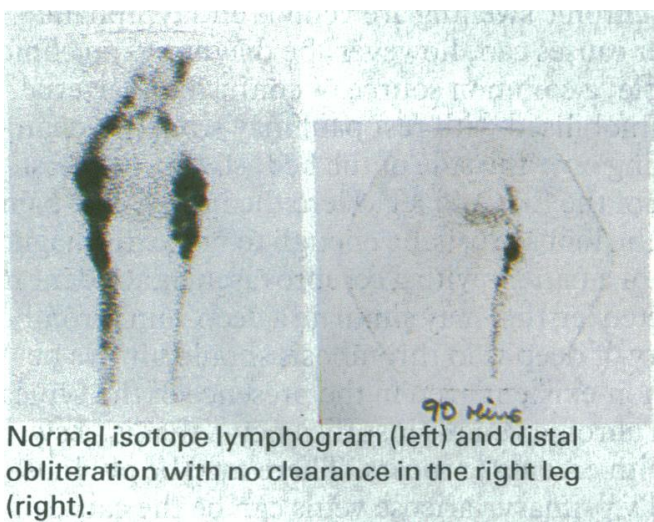

swelling and ulceration, but usually varicose veins in a patient with these chronic changes are incidental to deep venous obstruction or damage to the valves that is causing reflux. In the absence of postphlebitic changes-for example, in the aching fat leg - the presence of minor varicosities is unlikely to be of importance. Muscular cramps and aching legs are occasionally symptoms of venous insufficiency but are unreliable. If venous claudication is really present, a history of a progressively worsening "burning" pain on walking will be elicited.

The appropriate patients to refer for investigation of suspected chronic venous problems are those with obvious varicose veins, those with venous claudication, and -in particular - working people whose lives are made difficult by swelling, pain, or ulcers. Obese patients and those who have other problems such as cardiac failure and who spend most of their time immobile in a chair generally do not respond to treatment. In the vascular laboratory a range of investigations is available. The most useful of these are venous Doppler studies, venous pressure measurement, plethysmography, and duplex ultrasound scanning. These investigations can exclude a venous cause for the patient's problem or add functional information (such as the presence or absence of deep or superficial reflux or deep vein obstruction) and the newer duplex Doppler imaging system can show venous architecture and valve function.

The purpose of the investigations is to identify the subgroup of patients who will benefit from operations. The operations range from high ligation, stripping, and avulsion of varicose veins and subfascial ligation of perforators to the more complex operations for deep obstruction or reflux for which bypass grafting or vein and valve transplantation may be possible. Vein and valve transplantation is still being evaluated.

Most chronic venous problems are not amenable to surgical correction and conservative treatment is centred on adequate compression with specially fitted stockings, which need to be renewed roughly every six months. Below knee stockings are usually sufficient; full length stockings tend to roll down and "ruck" behind the knee.

Lymphatic causes - The huge "elephantiasis" leg of severe long standing lymphatic obstruction is obvious, but most patients present with unilateral painless oedema in a healthy looking leg in which there are no features of venous insufficiency. Most of these patients are young women with mild oedema and their prognosis is good; the oedema is unlikely to progress to the thigh and adequate conservative treatment should suffice. There is a subgroup of patients, however, who present with a more aggressive onset of oedema affecting both calf and thigh; these patients are likely to have patent distal lymphatics but ilioinguinal obstruction, and are the group who may benefit from early investigation with a view to mesenteric bridge lymphatic bypass. Good results are possible if this is done before obliteration of the distal lymphatics has occurred. A few may have an associated capillary naevus; this is indicative of megalymphatics that may also respond to surgical intervention. Cutaneous lymph or chylous vesicles are a sign of lymphatic reflux, and patients who have these incompetent reluxing lymphatics may have associated chylous ascites, chylothorax, or-more rarely-chylometrorrhoea, chyluria, or malabsorption. Ligation of the relevant lymphatics can produce immediate relief of symptoms.

The diagnosis of lymphoedema is often a diagnosis of exclusion except in a severe case. The indications for further investigation of the lymphatic system are: $(a)$ when the diagnosis is in doubt, and $(b)$ if ilioinguinal obstruction is suspected. Investigation is usually by a colloid labelled with a radioisotope.

Radionuclide lymphography uses high energy emitters bound to colloids which are taken up by the lymphatic vessels; it has the advantages of safety and a lower dose of radiation, and there is also the opportunity to carry out studies of clearance rates, which may provide useful functional information.

For bipedal lymphography the lymphatic vessels in the dorsum of the foot have firstly to be identified by injection of a diffusible dye into the web space, and then dissected out and cannulated under magnification. Radioopaque ethiodised oil (Lipiodol) is then injected and its progress followed radiologically. This is time consuming, and there is always the danger of pulmonary or cerebral oil embolism. If it is impossible to find peripheral lymphatic vessels to cannulate, the diagnosis of distal obliteration is confirmed. Megalymphatics or inguinal obstruction may also be seen. 


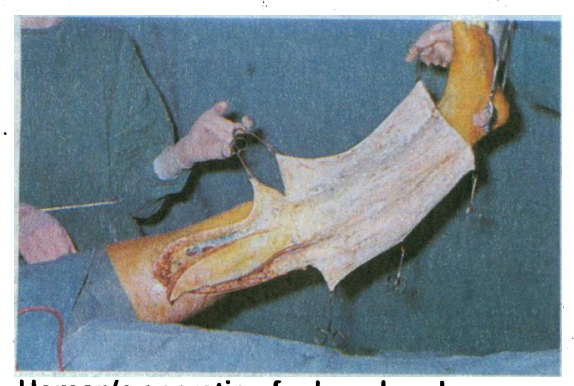

Homan's operation for lymphoedema.
It is not usually possible to cure lymphoedema but an active and enthusiastic approach can achieve great benefit. Most patients require only conservative treatment but if this is rigorously applied the results can be good. A short period of treatment in hospital using a Lymphapress can alter the patient's perception of the leg and engender enthusiasm for self treatment; cellulitis must be avoided by rigorous attention to hygiene.

A few patients benefit from operation, but this must never be carried out for cosmetic reasons. Those with heavy cumbersome legs can have the volume of subcutaneous tissue removed (Homan's operation) and the results are usually good if the indications for operation are correct. Such operations may also permit support hosiery to fit better.

A small group may benefit from operations to relieve lymphatic obstruction (mesenteric ileal bridging operation or direct lymphovenous anastomosis) but this group do not impinge on the mass of patients who should be treated conservatively.

The diagram of distal obliteration and pelvic obstruction are reproduced by permission of the publisher, Butterworth Heinemann, from DeWeese J, ed. Vascular surgery. In: Dudley H, Carter D, eds. Rob and Smith's operative surgery. 4th ed. 1985, 361 .

We acknowledge with thanks the assistance of the audiovisual department, St Mary's Hospital, London, in the preparation of the illustrations.

Mr D T Reilly, FRCs, is consultant surgeon, Watford General Hospital, Watford, and Mr John H N Wolfe, FRCS, is consultant vascular surgeon, St Mary's Hospital, London.

The ABC of Vascular Diseases has been edited by Mr John H N Wolfe.
Adipsic hypothalamic diabetes insipidus is a rare but life threatening complication of surgical clipping of anterior communicating artery aneurysms

Department of Medicine, Royal Infirmary, Edinburgh EM3 9YW

Bryan McIver, PHD, registrar in medicine

Chris Thompson, MD,

lecturer in medicine

Department of Medicine and Department of Clinical Neurosciences, Western General Hospital,

Edinburgh

Alan Connacher, MRCP, lecturer in medicine

Ian Whittle, MD, senior

lecturer in neurosurgery

Endocrine Unit, Royal Victoria Infirmary,

Newcastle upon Tyne NE1 4LP

Peter Baylis, MD, professor of clinical medicine

Correspondence to: Dr Chris Thompson, Department of Physiology, University of California, San Francisco, California 94143-0444, US.

\title{
Lesson of the Week
}

\section{Adipsic hypothalamic diabetes insipidus after clipping of anterior communicating artery aneurysm}

\author{
Bryan McIver, Alan Connacher, Ian Whittle, Peter Baylis, Chris Thompson
}

The development of inappropriate antidiuresis after subarachnoid haemorrhage from an intracranial arterial aneurysm is well recognised,,$^{1-3}$ with $5-10 \%$ of patients developing hyponatraemia. ${ }^{4}$ In contrast the occurrence of hypothalamic diabetes insipidus with the rupture of cerebral artery aneurysms is less well described ${ }^{5}$ and has an estimated incidence of only $0.04 \% .^{6}$ We report on two patients in whom severe hypothalamic diabetes insipidus occurred after clipping of a ruptured aneurysm in an anterior communicating artery. In each case there was a delay in diagnosis due to the absence of thirst and increased fluid intake, which are usually cardinal clinical features of diabetes insipidus, with consequent development of life threatening hypernatraemia.

\section{Case reports}

Case 1

A 39 year old woman developed sudden onset of severe frontal headache and vomiting. She had no history of headaches and no significant medical history. She was referred to hospital three days later because of persisting headaches. Clinical examination showed no focal neurological deficit, with a Glasgow coma scale rating of 15 , and blood pressure of $100 / 72 \mathrm{~mm} \mathrm{Hg}$. Lumbar puncture showed xanthochromic cerebrospinal fluid. She was treated with nimodipine $60 \mathrm{mg}$ four times daily. Plasma sodium concentration was $136 \mathrm{mmol} / \mathrm{l}$ (normal range $132-144 \mathrm{mmol} / \mathrm{l}$ ). Two days later she had further sudden frontal headaches, and computed tomography showed a small frontal interhemispheric haematoma and cerebral angiography showed an aneurysm in an anterior communicating artery.

The aneurysm was successfully clipped through a left pterional craniotomy. During clip placement, however, the aneurysm fundus ruptured. She was artificially ventilated for one day in the intensive care unit. On return to the neurosurgical unit she was noted to have hypernatraemia (plasma sodium concentration $155 \mathrm{mmol} / \mathrm{l}$ ) and polyuria (urine volume $>300 \mathrm{ml} / \mathrm{h}$ ) with a urine specific gravity of 1005 , though she did not complain of thirst. After impaired consciousness associated with tachycardia and hypotension further computed tomography showed evidence of intraventricular haemorrhage, moderate hydrocephalus, and an infarction in the right anterior cerebral artery territory. She rapidly improved after a burrhole insertion of a cerebrospinal fluid access device but stil had polyuria and hypernatraemia with minimal fluid intake. The polyuria responded to intramuscular desmopressin, and she was thereafter managed with intranasal desmopressin twice daily and a fixed fluid intake of two litres a day. Because of poor compliance with oral fluid intake plasma osmolality fluctuated from $290 \mathrm{mmol} / \mathrm{kg}$ to $330 \mathrm{mmol} / \mathrm{kg}$ (normal range 282 $298 \mathrm{mmol} / \mathrm{kg}$ ). One month postoperatively she received an infusion of hypertonic $(855 \mathrm{mmol} / \mathrm{l})$ sodium chloride at $0.06 \mathrm{ml} / \mathrm{kg} / \mathrm{min}$ for two hours.

Despite pronounced hyperosmolality plasma vasopressin concentrations remained inappropriately low, indicative of hypothalamic diabetes indipidus (figure); and thirst ratings, measured on a visua analogue scale $^{89}$ remained unchanged. In the two hours after the infusion she drank $175 \mathrm{ml}$ of water. Anterior pituitary function was normal. Six month postoperatively her diabetes insipidus was treated with desmopressin twice daily, but because of adipsia she required a fixed fluid intake of two litres daily, with close supervision to monitor weight and plasma biochemistry and to encourage fluid intake. 